\title{
Efficient Transient Simulation of Single-Phase Grid-Feeding Converter System Using Frequency Response Optimized Integrators Considering Second Order Derivative
}

This paper was downloaded from TechRxiv (https://www.techrxiv.org).

\section{LICENSE}

CC BY 4.0

SUBMISSION DATE / POSTED DATE

$20-02-2021 / 24-02-2021$

\section{CITATION}

Lei, Sheng; Flueck, Alexander (2021): Efficient Transient Simulation of Single-Phase Grid-Feeding Converter System Using Frequency Response Optimized Integrators Considering Second Order Derivative. TechRxiv. Preprint. https://doi.org/10.36227/techrxiv.14069090.v1

$\mathrm{DOI}$ 
(C) 2021 IEEE. Personal use of this material is permitted. Permission from IEEE must be obtained for all other uses, in any current or future media, including reprinting/republishing this material for advertising or promotional purposes, creating new collective works, for resale or redistribution to servers or lists, or reuse of any copyrighted component of this work in other works.

This paper has been accepted by the 2021 IEEE PES Innovative Smart Grid Technologies Conference. 


\title{
Efficient Transient Simulation of Single-Phase Grid- Feeding Converter System Using Frequency Response Optimized Integrators Considering Second Order Derivative
}

\author{
Sheng Lei ${ }^{1,2}$, Student Member, IEEE, and Alexander Flueck ${ }^{1}$, Senior Member, IEEE \\ ${ }^{1}$ Department of Electrical and Computer Engineering \\ Illinois Institute of Technology \\ Chicago, IL, USA \\ ${ }^{2}$ Mathematics and Computer Science Division \\ Argonne National Laboratory \\ Lemont, IL, USA
}

Email: slei3@hawk.iit.edu, and flueck@iit.edu

\begin{abstract}
A novel power system transient simulation scheme based on frequency response optimized integrators considering second order derivative is applied to a single-phase grid-feeding converter system in this paper. The converter system under study is introduced in detail as well as how the different numerical integrators are utilized for each constituting component. Numerical case studies show that the novel transient simulation scheme is able to achieve computational efficiency without sacrificing accuracy.
\end{abstract}

Index Terms-Distributed energy resource (DER), frequency response optimized integrator, grid converter control, singlephase system, transient simulation.

\section{INTRODUCTION}

The recent years have witnessed an increasing amount of single-phase grid-feeding converter systems integrated to power grids, mainly for exploitation of small-scale renewable energy resources and interconnection between electric vehicles and utility networks [1]-[2]. It is thus of importance to study power system stability as impacted by these devices, in order to guarantee secure operation of the systems. To this end, electromagnetic transient (EMT) simulation is a powerful tool. It models individual phases in detail and is able to trace comprehensive time domain dynamics of the systems [3]-[4].

Nevertheless the traditional EMT simulation is computationally inefficient for stability studies. It requires unnecessarily small step sizes during simulation runs, due to the presence of the utility frequency $(50$ or $60 \mathrm{~Hz})$ in voltage and current waveforms [5]-[6]. Otherwise the numerical integrators adopted, such as the implicit trapezoidal method, will introduce significant error or even distort the results with large step sizes [5]-[6].

In a recent work of the authors [7], a novel power system transient simulation scheme was put forward based on frequency response optimized integrators considering second order derivative. The error introduced by the numerical integrators is minimized according to the utility frequency of the studied system so that computational efficiency is improved by using large step sizes without sacrificing accuracy. It is shown that the novel transient simulation scheme is especially suitable for stability studies on general unbalanced power systems.

In this paper, the novel transient simulation scheme is applied to a typical single-phase grid-feeding converter system. Individual components of the converter system and how the frequency response optimized integrators considering second order derivative are utilized are both explained in detail. It is found that the novel transient simulation scheme is able to achieve significant efficiency while maintaining satisfactory accuracy as applied to this type of device. The rest of this paper is organized as follow. Section II reviews the novel transient simulation scheme. The converter system under study is introduced in Section III, along with how the numerical integrators are applied to the individual components. Section IV verifies the accuracy and efficiency of the novel transient simulation scheme as applied to the converter system via numerical case studies. Section V concludes the paper.

\section{REVIEW OF THE NOVEL TRANSIENT SIMULATION SCHEME}

\section{A. Frequency Response Optimized Integrators Considering Second Order Derivative}

A power system is typically modeled as a set of differential-algebraic equations (DAEs) for analysis [3]-[4]. A transient simulation scheme seeks numerical solutions to the equation set, given system parameters and initial conditions. The differential equations involved are discretized and converted into algebraic ones by certain numerical integrators to be solved numerically [3]-[4]. Commonly used numerical 
integrators include the implicit trapezoidal method and the backward Euler method [8].

Consider a general differential equation

$$
\dot{x}=f(t, x, u)
$$

where $x$ is the state variable; $t$ is the time instant; $u$ is the input; $f$ is a function depending on $t, x$ and $u$. To be numerically solved, (1) may be discretized by a numerical integrator considering second order derivative as

$$
x_{t}=x_{t-h}+b_{0} \dot{x}_{t}+b_{-1} \dot{x}_{t-h}+c_{0} \ddot{x}_{t}+c_{-1} \ddot{x}_{t-h}
$$

where $h$ is the step size; $b_{0}, b_{-1}, c_{0}$ and $c_{-1}$ are coefficients to be determined. A specific selection for these coefficients determines a numerical integrator. Note that the second order derivative of the state variable is calculated as

$$
\ddot{x}=\frac{\partial f}{\partial t}+\frac{\partial f}{\partial x} \dot{x}+\frac{\partial f}{\partial u} \dot{u}
$$

Clearly information about the derivative of the input is required. Such information may be externally given or numerically calculated according to the input.

The coefficients in (2) can be chosen so that the resulting numerical integrator introduces no error at a specified angular frequency $\omega_{\text {select }}$ despite the specific value of the step size. In certain situations, the state variable $x$ in (1) and (2) has a dominant frequency component. For example, the voltage and current waveforms in power systems are dominated by the utility frequency. Accordingly $\omega_{\text {select }}$ can be specified at the dominant frequency to significantly reduce the error introduced by the discretization process. The frequency response of the resulting numerical integrator is optimized in this sense. As a result, large step sizes are enabled to increase efficiency without sacrificing accuracy.

Coefficients for four frequency response optimized integrators considering second order derivative defined in [7], namely Integrators A-D, are listed in Table I. Integrators A and $\mathrm{B}$ are accurate for state variables dominated by the frequency components at $\omega_{\text {select }}$ and $0 \mathrm{~Hz}$. Integrators $\mathrm{C}$ and D are accurate for slow variants. Integrators $\mathrm{A}$ and $\mathrm{C}$ are more accurate than B and D respectively. Derivation and detailed discussion of these numerical integrators can be found in [7].

TABLE I. FREQUENCY RESPONASE OPTIMIZED INTERATORS CONSIDERING SECOND ORDER DERIVATIVE

\begin{tabular}{|c|c|c|c|c|}
\hline \multirow{2}{*}{ Coefficient } & A & Numerical Integrator & B & D \\
\cline { 2 - 5 } & $\frac{h}{2}$ & $\frac{\sin \left(\omega_{\text {select }} h\right)}{\omega_{\text {select }}}$ & $\frac{h}{2}$ & $h$ \\
\hline$b_{0}$ & $\frac{h}{2}$ & 0 & $\frac{h}{2}$ & 0 \\
\hline$b_{-1}$ & $\frac{-1}{\omega_{\text {select }}^{2}}+\frac{h}{2 \omega_{\text {select }}} \cot \left(\frac{\omega_{\text {select }} h}{2}\right)$ & $\frac{\cos \left(\omega_{\text {select }} h\right)-1}{\omega_{\text {select }}^{2}}$ & $-\frac{h^{2}}{12}$ & $-\frac{h^{2}}{2}$ \\
\hline$c_{0}$ & $\frac{1}{\omega_{\text {select }}^{2}}-\frac{h}{2 \omega_{\text {select }}} \cot \left(\frac{\omega_{\text {select }} h}{2}\right)$ & 0 & $\frac{h^{2}}{12}$ & 0 \\
\hline$c_{-1}$ & 0 & & \\
\hline
\end{tabular}

\section{B. The Novel Transient Simulation Scheme}

The novel transient simulation scheme discretizes the differential equations of the studied system with multiple frequency response optimized integrators considering second order derivative according to the dominant frequency components of the state variables. Integrator $\mathrm{A}$ is used for the differential equations of which the state variable is dominated by the utility frequency while Integrator $C$ for those of which the state variable is a slow variant.

A fixed step size is adopted by the simulation scheme during a simulation run, which is the same as commonly used in power system transient simulation. The equations of power system network and other power system devices are solved simultaneously via iteration at each time step. Consistency of the whole system is thus ensured to achieve high fidelity. Discontinuity events such as faults and breaker switching are properly dealt with via a similar technique to the mature Critical Damping Adjustment (CDA) [9]. Integrators B and D are used as an alternative to Integrators $A$ and $C$ respectively for these events.

\section{SINGLE-PHASE GRID-FEEDING CONVERTER SYSTEM}

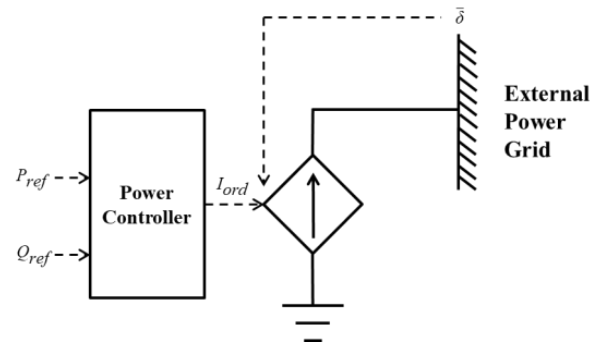

Figure 1. Sketch diagram of the converter system

In this section, the single-phase grid-feeding converter system studied in this paper is introduced. The structure of the converter system is based on [10]. Fig. 1 shows a sketch diagram of the converter system. The main objective of the converter system is to regulate its real and reactive power output. The power controller receives real and reactive power references and generates control orders for the remaining part of the converter system, the overall behavior of which can be understood as an equivalent controlled AC current source. As the current order generated by the power controller is typically expressed as a phasor in the device reference frame, the cumulative phase angle of the voltage at the terminal of the converter system has to be provided so that the equivalent controlled AC current source is able to generate the correct current waveform.

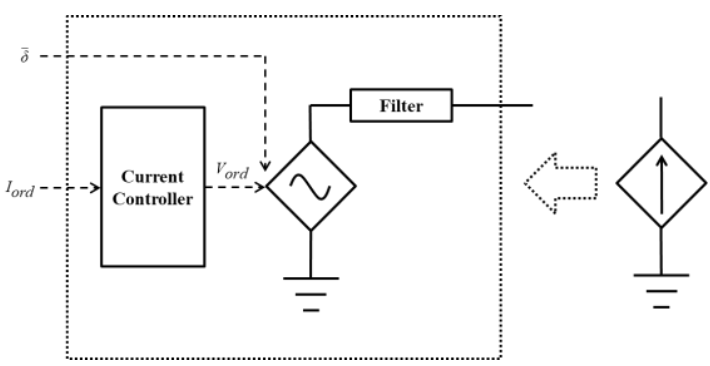

Figure 2. Equivalent controlled AC current source.

The equivalent controlled AC current source consists of a filter, a voltage source converter (VSC) and related controllers, as shown in Fig. 2. A current controller receives the current orders from the power controller and generates voltage orders which also form a phasor in the device reference frame. Taking into account the voltage order phasor and the 
cumulative phase angle of the terminal voltage, the VSC generates the required voltage waveform. The output waveform of the VSC goes through a filter so that the final output is close to a sinusoid at the utility frequency.

As the phasor representation is used by the controllers, corresponding voltage and current waveforms have to be measured and converted into phasors. As the cumulative phase angle of the voltage is required at the terminal of the converter system, a synchronization algorithm [11] is needed. In addition, real and reactive powers are to be measured. Such functionalities are performed by a measurement and synchronization mechanism.

Individual constituting components of the converter system are detailed as follows along with how the numerical integrators reviewed in Subsection II A are applied.

\section{A. L Filter}

For a grid-feeding converter system, the filter is typically an inductor (L filter) [10], [12]. The transient model is

$$
\frac{d i_{o}}{d t}=\frac{1}{L_{f}}\left(v_{i}-v_{o}\right)
$$

where $i_{o}$ is the current through the $\mathrm{L}$ filter; $L_{f}$ is the inductance of the L filter; $v_{i}$ is the output voltage of the VSC; $v_{o}$ is the terminal voltage of the converter system. Waveforms within the $\mathrm{L}$ filter are dominated by sinusoids at the utility frequency. Therefore Integrator A is used for the differential equation.

\section{B. $V S C$}

In power system stability studies, a VSC including the pulse-width modulation (PWM) is usually simplified as a controlled AC voltage source [13]-[14]. The DC side is assumed to be strong enough to guarantee the required functionalities of the VSC and is not further considered. Receiving voltage order phasor in the device reference frame and cumulative phase angle of the terminal voltage, the controlled AC voltage source accordingly generates the output voltage waveform

$$
v_{i}=V_{\text {ord }, m} \cos \left(\bar{\delta}+V_{\text {ord }, a, d e v}\right)
$$

where $V_{o r d, m}$ is the voltage order phasor magnitude; $V_{\text {ord, a, dev }}$ is the voltage order phasor angle in the device reference frame; $\bar{\delta}$ is the cumulative phase angle of the terminal voltage. Note that they are time-varying. The VSC is a pure algebraic component in that no differential equation is involved. Hence no numerical integrator is needed.

\section{Power Controller}

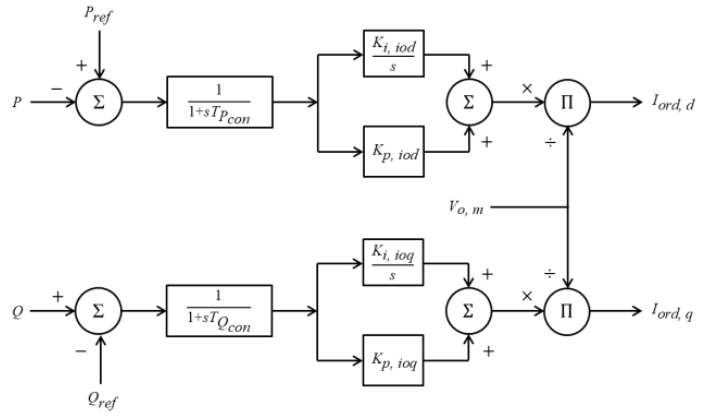

Figure 3. Power controller.
Fig. 3 shows a design of a power controller [12]. It receives externally given real and reactive power references and generates current orders. The current orders form a phasor in the device reference frame. Besides the power references, the power controller also requires real power output, reactive power output and terminal voltage phasor magnitude of the converter system as its inputs. Integrator $\mathrm{C}$ is used for the differential equations of the power controller as the signals involved are basically slow variants.

\section{Current Controller}

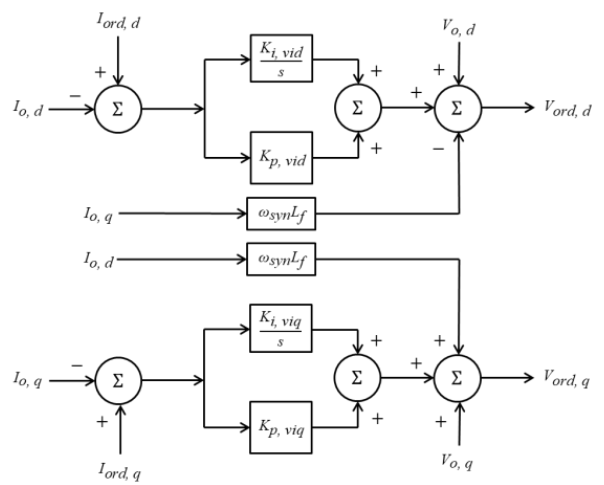

Figure 4. Current controller.

Fig. 4 shows a design of a current controller [10], [12], [15]. It receives current orders generated by the power controller and generates voltage orders. Again the voltage orders form a phasor in the device reference frame. Besides the current orders, the current controller also requires the current phasor through the $\mathrm{L}$ filter and the terminal voltage phasor as its input. Integrator $\mathrm{C}$ is used for the current controller.

\section{E. Measurement and Synchronization Mechanism}

1) In-phase and quadrature signal generation: An improved second-order generalized integrator based quadrature signal generator (SOGI-QSG) [11] may be used to generate the required in-phase and quadrature signals. The improved SOGI-QSG is shown in Fig. 5. It receives a signal $x$ as its input; and it outputs the in-phase signal which approximates the fundamental frequency component of $x$ and the quadrature signal which approximates a sinusoidal signal that lags the fundamental frequency component of $x$ by $\pi / 2$. DC offset and high frequency components are suppressed in the in-phase and quadrature signals due to the frequency response of the improved SOGI-QSG. Besides the signal $x$, it also requires the fundamental angular frequency. Integrator $\mathrm{A}$ is used for the improved SOGI-QSG.

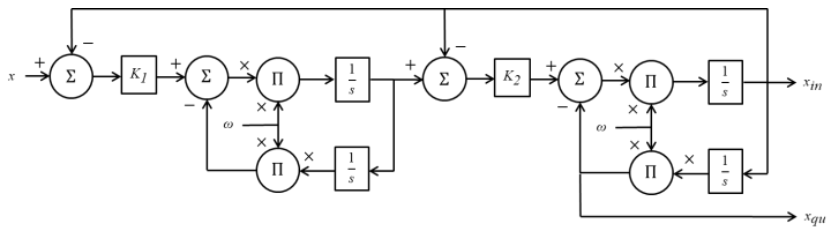

Figure 5. Improved SOGI-QSG.

2) Phase shift: A phase shift factor is applied to the inphase and quadrature signals to generate the real and 
imaginary parts of the corresponding phasor in the device reference frame

$$
X=X_{d}+j X_{q}=e^{-j \bar{\delta}}\left(x_{i n}+j x_{q u}\right)
$$

Equivalently

$$
\begin{gathered}
X_{d}=\cos (\bar{\delta}) x_{i n}+\sin (\bar{\delta}) x_{q u} \\
X_{q}=-\sin (\bar{\delta}) x_{i n}+\cos (\bar{\delta}) x_{q u}
\end{gathered}
$$

This is a pure algebraic component so no numerical integrator is needed.

3) Phase-locked loop (PLL): Fig. 6 shows a PLL [10][11]. The imaginary part of the terminal voltage phasor is fed into the PLL. The output is the cumulative phase angle of the terminal voltage. In transient simulation, an alternative but equivalent implementation can be adopted, where the phase angle regarding the synchronously rotating referece frame is calculated, which is also understood as the phasor angle. Note that the phasor angle is time-varying unless the system is in the steady state. The cumulative phase angle is then calculated as

$$
\bar{\delta}=\omega_{s y n} t+\delta
$$

where $\omega_{\text {syn }}$ is the synchronous angular frequency; $\delta$ is the phasor angle. Integrator $\mathrm{C}$ is used for the PLL.

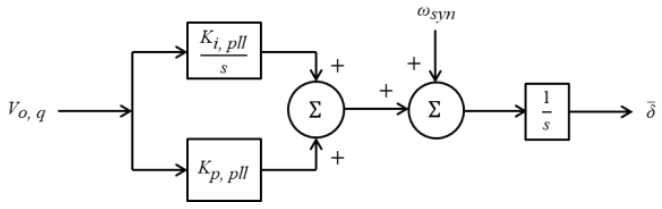

Figure 6. PLL.

4) Real power output, reactive power output and terminal voltage phasor magnitude measurements: The real power output, reactive power output and terminal voltage phasor magnitude are pre-calculated as [15]

$$
\begin{gathered}
P_{p r e}=V_{o, d} I_{o, d}+V_{o, q} I_{o, q} \\
Q_{p r e}=-V_{o, d} I_{o, q}+V_{o, q} I_{o, d} \\
V_{o, m, p r e}=\sqrt{{V_{o, d}{ }^{2}+V_{o, q}^{2}}^{2}}
\end{gathered}
$$

where $P_{\text {pre }}, Q_{\text {pre }}$ and $V_{o, m}$, pre are the pre-calculated real power output, reactive power output and terminal voltage phasor magnitude. The measured values are obtained by passing the pre-calculated values to low-pass filters [15]

$$
\begin{gathered}
\dot{P}=\frac{1}{T_{P}}\left(-P+P_{p r e}\right) \\
\dot{Q}=\frac{1}{T_{Q}}\left(-Q+Q_{p r e}\right) \\
\dot{V}_{o, m}=\frac{1}{T_{V}}\left(-V_{o, m}+V_{o, m, p r e}\right)
\end{gathered}
$$

where $T_{P}, T_{Q}$ and $T_{V}$ are time constants of the low-pass filters; $P, Q$ and $V_{o, m}$ are the measured real power output, reactive power output and terminal voltage phasor magnitude respectively. Integrator $\mathrm{C}$ is used for these measurements.

\section{NUMERICAl CASE STUdIES}

The single-phase grid-feeding converter system has been included in the MATLAB implementation of the novel transient simulation scheme [7]. The accuracy and efficiency of the simulation scheme regarding this type of device is to be verified via numerical case studies in this section, by comparing the results from and the time consumption of the simulation scheme to those obtained from an iterative EMT simulator [7] applied to the same test system. The iterative EMT simulator is also implemented with MATLAB, adopting the same structure and execution as the novel transient simulation scheme, except that differential equations are discretized with the implicit trapezoidal method for normal time steps and the backward Euler method for discontinuities. As the only difference between the two simulation schemes is in the numerical integrators and the resulting specific implementation, the comparison is fair.

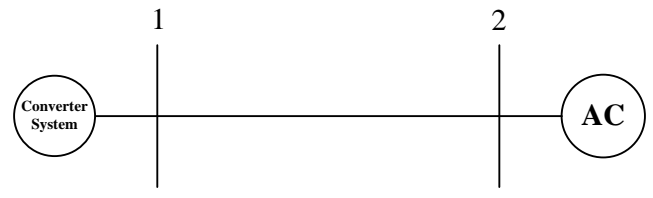

Figure 7. A 2-bus power system.

Numerical case studies are performed on a 2-bus power system as shown in Fig. 7. Both busses are a single-phase one. A branch connects the two busses, of which the impedance is $0.1586+j 0.1608$. An ideal AC voltage source is connected to

\begin{tabular}{|c|c|c|c|}
\hline \multicolumn{2}{|c|}{ L Filter } & \multicolumn{2}{|c|}{ Current Controller } \\
\hline$L_{f}$ & $2.1008 \times 10^{-4}$ & $K_{p, v i q}$ & 0.25 \\
\hline \multicolumn{2}{|c|}{ Power Controller } & \multirow{2}{*}{\multicolumn{2}{|c|}{$\begin{array}{c}\text { Measurement and } \\
\text { Synchronization Mechanism }\end{array}$}} \\
\hline$T_{P_{c o n}}$ & 0.1 & & \\
\hline$K_{i, \text { iod }}$ & 10 & $K_{1, v o}$ & 1.5 \\
\hline$K_{p, \text { iod }}$ & 1.3 & $K_{2, v o}$ & 3 \\
\hline$T_{Q_{c o n}}$ & 0.1 & $K_{1, \text { io }}$ & 1.5 \\
\hline$K_{i, i o q}$ & 10 & $K_{2, \text { io }}$ & 3 \\
\hline$K_{p, \text { ioq }}$ & 1.3 & $K_{i, p l l}$ & 5000 \\
\hline \multicolumn{2}{|c|}{ Current Controller } & $K_{p, p l l}$ & 100 \\
\hline$K_{i, v i d}$ & 0.01 & $T_{P}$ & 0.02 \\
\hline$K_{p, v i d}$ & 0.25 & $T_{Q}$ & 0.02 \\
\hline$K_{i, v i q}$ & 0.01 & $T_{V}$ & 0.02 \\
\hline
\end{tabular}
Bus 2; the voltage phasor magnitude is 1.0 while the voltage phasor angle is $-\pi / 2$ rad. A single-phase grid-feeding converter system is connected to Bus 1 ; the real power output is 0.3 while the reactive power output is 0.12. Dynamic parameters of the converter system are listed in Table II. Simulation runs start from the steady state. At $0.1 \mathrm{~s}$, a fault is applied at Bus 1 with a fault resistance of 0.1 p.u.. At $0.4 \mathrm{~s}$, the fault is cleared.

TABLE II. DYNAMIC PARAMETERS OF THE CONVERTER SYSTEM

\section{A. Qualitative Comparison}

Results from the iterative EMT simulator with a step size of $5 \mu \mathrm{s}$ are used as the reference. Fig. 8 shows the real power output of the converter system. Fast transients are observed immediately after the fault application and clearance. Results from the novel transient simulation scheme and the iterative EMT simulator with larger step sizes are compared to the reference to observe their accuracy. For better visualization, only the results around $0.5 \mathrm{~s}$ are presented. Note that the novel transient simulation scheme gives more accurate results than the iterative EMT simulator with the same or one-half the step 
size. It is thus possible to use the novel transient simulation scheme with large step sizes to reduce the time consumption while maintaining satisfactory accuracy.

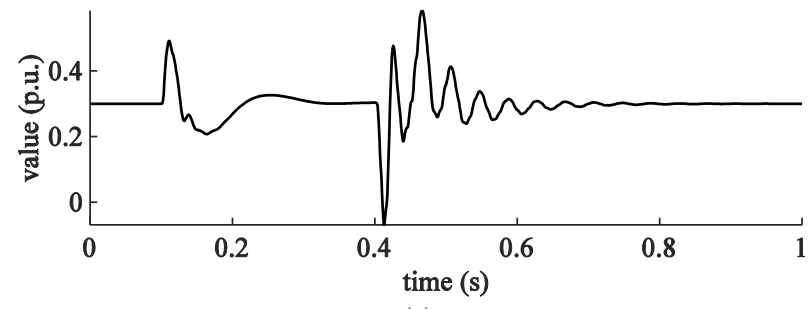

(a)

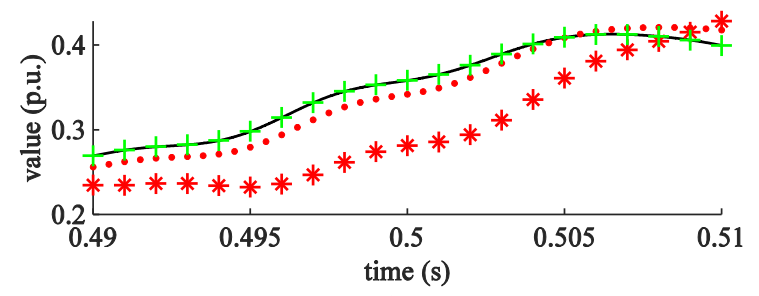

(b)

Figure 8. Real power output. (a) Reference. (b) Comparison to the reference Black solid line: the reference. Green plus sign markers: the novel transient simulation scheme with a $1000 \mu$ s step size. Red point markers: the iterative EMT simulator with a $500 \mu$ s step size. Red asterisk markers: the iterative EMT simulator with a $1000 \mu$ s step size.

\section{B. Quantitative Comparison}

This paper uses the following error measurement to quantitatively study the numerical error of the simulation schemes. Suppose that $x$ is a variable to be considered. The relative error with respective to $x$ of a simulation scheme given a specific step size is

$$
\operatorname{err}(x)=\left\|x_{\text {com }}-x_{\text {ref }}\right\|_{2} /\left\|x_{\text {ref }}\right\|_{2} \times 100
$$

where $x_{\text {com }}$ is the computed value from a simulation scheme; $x_{\text {ref }}$ is the reference value. The 2-norm is calculated at common time instants of $x_{\text {com }}$ and $x_{\text {ref }}$.

The error measurement (16) is applied to Bus 1 voltage waveform and Bus 1 voltage phasor angle. The resulting two errors are referred to as voltage error and phase error respectively for simplicity. Voltage error and phase error of the novel transient simulation scheme and the iterative EMT simulator with different step sizes are listed in Table III, along with their time consumption. The duration of the simulation runs in Table III is from $0.0 \mathrm{~s}$ to $2.0 \mathrm{~s}$.

TABLE III. COMPARISON OF THE NOVEL TRANSIENT SIMULATION SCHEME AND THE ITERATIVE EMT SIMULATOR

\begin{tabular}{|c|c|c|c|c|c|c|}
\hline \multirow{2}{*}{$\begin{array}{c}\text { Step Size } \\
(\boldsymbol{\mu s})\end{array}$} & \multicolumn{2}{|c|}{$\begin{array}{c}\text { Time Consumption } \\
(\mathbf{s})\end{array}$} & \multicolumn{2}{c|}{ Voltage Error } & \multicolumn{2}{c|}{ Phase Error } \\
\cline { 2 - 7 } & Novel & EMT & Novel & EMT & Novel & EMT \\
\hline 125 & 46.21 & 36.85 & 0.0029 & 0.0613 & 0.0015 & 0.0292 \\
\hline 250 & 24.29 & 18.73 & 0.0059 & 0.2487 & 0.0031 & 0.1188 \\
\hline 500 & 13.09 & 9.71 & 0.0123 & 1.0249 & 0.0063 & 0.4897 \\
\hline 1000 & 7.11 & 5.79 & 0.0368 & 4.3504 & 0.0154 & 2.1011 \\
\hline 2000 & 3.98 & 3.46 & 0.1808 & 16.0825 & 0.0794 & 8.8026 \\
\hline 4000 & 2.22 & 2.93 & 1.6557 & 127.0 & 0.7287 & 18244 \\
\hline
\end{tabular}

As can be seen from Table III, with the same step size, the novel transient simulation scheme typically requires more time than the iterative EMT simulator, but less than twice as much. The higher cost is due to the consideration of second order derivative by the numerical integrators. Nevertheless the increased cost pays off with much higher accuracy which is at least more than 4 times compared to the iterative EMT simulator. Therefore the novel transient simulation scheme obtains similar or more accurate results with less time consumption.

\section{CONCLUSION}

Single-phase grid-feeding converter system has been included into the novel transient simulation scheme based on frequency response optimized integrators considering second order derivative. Constituting components of the converter system and how they are implemented in the simulation scheme are explained in detail. Results show that efficiency and accuracy are simultaneously achieved by the simulation scheme as applied to the studied converter system.

\section{REFERENCES}

[1] M. Pahlevani and P. Jain, "A Fast DC-Bus Voltage Controller for Bidirectional Single-Phase AC/DC Converters," in IEEE Transactions on Power Electronics, vol. 30, no. 8, pp. 4536-4547, Aug. 2015.

[2] D. B. W. Abeywardana, B. Hredzak and V. G. Agelidis, "An Input Current Feedback Method to Mitigate the DC-Side Low-Frequency Ripple Current in a Single-Phase Boost Inverter," in IEEE Transactions on Power Electronics, vol. 31, no. 6, pp. 4594-4603, June 2016.

[3] H. W. Dommel, EMTP Theory Book. Vancouver, BC, Canada: Microtran Power System Analysis Corporation, May 1992.

[4] N. Watson and J. Arrillaga, Power System Electromagnetic Transient Simulation. London, U.K.: The Institution of Electrical Engineers, 2003.

[5] F. Gao and K. Strunz, "Frequency-Adaptive Power System Modeling for Multiscale Simulation of Transients," in IEEE Transactions on Power Systems, vol. 24, no. 2, pp. 561-571, May 2009.

[6] P. Zhang, J. R. Marti and H. W. Dommel, "Synchronous Machine Modeling Based on Shifted Frequency Analysis," in IEEE Transactions on Power Systems, vol. 22, no. 3, pp. 1139-1147, Aug. 2007.

[7] S. Lei and A. Flueck, "Efficient Power System Transient Simulation Based on Frequency Response Optimized Integrators Considering Second Order Derivative," presented at the 2020 IEEE PES General Meeting. [Online]. Available: https://arxiv.org/abs/2005.00964

[8] R. M. Corless and N. Fillion, A Graduate Introduction to Numerical Methods. New York, NY, USA: Springer-Verlag, 2013.

[9] J. R. Marti and J. Lin, "Suppression of numerical oscillations in the EMTP power systems," in IEEE Transactions on Power Systems, vol. 4, no. 2, pp. 739-747, May 1989.

[10] J. Rocabert, A. Luna, F. Blaabjerg and P. Rodríguez, "Control of Power Converters in AC Microgrids," in IEEE Transactions on Power Electronics, vol. 27, no. 11, pp. 4734-4749, Nov. 2012.

[11] S. Golestan, J. M. Guerrero and J. C. Vasquez, "Single-Phase PLLs: A Review of Recent Advances," in IEEE Transactions on Power Electronics, vol. 32, no. 12, pp. 9013-9030, Dec. 2017.

[12] E. Muljadi, M. Singh and V. Gevorgian, "User guide for PV dynamic model simulation written on PSCAD platform", Nov. 2014.

[13] A. A. van der Meer, M. Gibescu, M. A. M. M. van der Meijden, W. L. Kling and J. A. Ferreira, "Advanced Hybrid Transient Stability and EMT Simulation for VSC-HVDC Systems," in IEEE Transactions on Power Delivery, vol. 30, no. 3, pp. 1057-1066, June 2015.

[14] W. Du et al., "Modeling of Grid-Forming and Grid-Following Inverters for Dynamic Simulation of Large-Scale Distribution Systems," in IEEE Transactions on Power Delivery.

[15] N. Pogaku, M. Prodanovic and T. C. Green, "Modeling, Analysis and Testing of Autonomous Operation of an Inverter-Based Microgrid," in IEEE Transactions on Power Electronics, vol. 22, no. 2, pp. 613-625, March 2007. 